\title{
Efficacy of Customized Foot Orthoses in Children Aged 6 - 7 Years Old with Symptomatic Flexible Flat Foot (SFFF)
}

\section{George Tsigaras', Vasileios Goulios², Sotiria Exadaktylou ${ }^{3}$, Thomas Besios ${ }^{4}$, Maria Milioudi1, Kostastantinos Chandolias ${ }^{5}$, Victoria Laspa ${ }^{5}$, Alexandra Xristara ${ }^{5}$}

\author{
${ }^{1}$ Hippokratio General Hospital, Thessaloniki, Greece \\ ${ }^{2}$ St Luke's Hospital, Thessaloniki, Greece \\ ${ }^{3}$ Papageorgiou General Hospital, Thessaloniki, Greece \\ ${ }^{4}$ Physiotherapy Department, University of Thessaly, Filellinon, Greece \\ ${ }^{5}$ Department of Physical Therapy, International University of Greece, Thessaloniki, Greece \\ Email:m.milioudi@yahoo.com
}

How to cite this paper: Tsigaras, G., Goulios, V., Exadaktylou, S., Besios, T., Milioudi, M., Chandolias, K., Laspa, V. and Xristara, A. (2020) Efficacy of Customized Foot Orthoses in Children Aged 6 - 7 Years Old with Symptomatic Flexible Flat Foot (SFFF). Open Journal of Preventive Medicine, 10 , 217-224.

https://doi.org/10.4236/ojpm.2020.108015

Received: June 26, 2020

Accepted: August 15, 2020

Published: August 18, 2020

Copyright () 2020 by author(s) and Scientific Research Publishing Inc. This work is licensed under the Creative Commons Attribution International License (CC BY 4.0).

http://creativecommons.org/licenses/by/4.0/

\section{(c) (i) Open Access}

\begin{abstract}
Introduction: Pediatric Flat Foot (PFF) is a deviation consisting of loss of the Medial Longitudinal Arch in children. Purpose: Aim of this study is to collect information on the recent literature and to investigate through clinical assessment and parental observations the effectiveness of the use of foot orthotics by children with SFFF. Method: 20 children aged $6-7$ years old with mobility difficulties were evaluated before and after a six-month treatment with orthotics regarding pain, post-game pain, fatigue during game, balance impairment, gait deviations, falls, clumsiness, activity avoidance and worn soles. Results: Study results indicate that foot orthotics (FOs) appears to have a positive impact on the advance of pain, post game pain, gait deviations and worn insoles. It is implied that orthoses have a great potential as a treatment for SFFF. Thorough comprehension of the literature evidence, as well as composition of supplementary studies of larger pediatric populations is essential in order to reach a consensus on the use of foot orthotics (FOs) by children SFFF.
\end{abstract}

\section{Keywords}

Efficacy, Orthoses, Flexible, Flatfoot, Children

\section{Introduction}

Pes planus is a deformation of the lower extremity in children. It is defined as a 
deviation consisting of loss of the Medial Longitudinal Arch (MLA) of the foot where it contracts or nearly contracts the ground. This deformity can be apparent in any age but it mostly affects children [1]. When it presents in childhood it is referred to as Pediatric Flat Foot (PFF).

Flat foot (FF), besides children, has been described in an abundance of cases from infancy through adolesence [2]. Pediatric flat foot (PFF) is reported in up to $20 \%$ of children and adolescents.

Howbeit, there is an ongoing debate whether it represents a normal variant or pathological feature. It is estimated that $10 \%-60 \%$ of children with pediatric flatfoot (PFF) complain about functional difficulties [3]. PFF is categorised according to anatomical or other aetiological factors. PFF could be due to constitutional deviation, isolated pathology or part of a clinical entity such as generalized ligamentous laxity, neurological and muscular abnormalities, genetic conditions, various syndromes and collagen vascular disease [4] [5] [6]

Pes planus either caused by genetic or neuromuscular disorders, as already mentioned, can be classified into flexible and rigid flatfoot. Flexible flatfoot is recognized by flattening of the Medial Longitudinal Arch (MLA) when weight-bearing, while MLA will be apparent when the patient stands on toes. Rigid flatfoot is identified by a stiff, flattened arch equally on and off weight-bearing [7] [8] [9] [10].

Another division of flatfoot concerning children is symptomatic and asymptomatic, taking into consideration the development and evolution of the arch. In the absence of universal specific recommendations on the appropriate diagnostic procedure of measurement and standardization of the arch, diagnosis is based on clinical examination, angle-drawing techniques, evaluation of weight, posture, construction of the arch and semiology of pain or fatigue [11]-[16].

We investigate the efficacy of utilization of customized orthotics on Symptomatic Flexible Flat Feet (SFFF) in children with the cooperation of their parents.

\section{Method}

A sample of thirty seven (37) children of both genders (boys and girls) aged 6 - 7 years old was examined from January 2018 to January 2020, of which twenty children (20) were eligible for the study.

Inclusion criteria for participation were hypermobile joints on clinical assessment scoring higher than 4 in Belghton test, symptoms of pain in the lower limbs and parental concern for the patients' functional performance. As exclusion criteria were determined the absence of pain in the lower extremities, history of trauma, surgery or diagnosed genetic, neurological or musculoskeletal disease.

A questionnaire and a participant consent form was provided to parents to note movement impairment and describe the general health status of the child. The foundation of the composition of the questionnaire was the constant reporting from the caregivers of the deviations of past years along with the clinical 
experience of the multidisciplinary team of experts. Pain, falls, activity avoidance, fatigue and worn soles were detected. All participants were examined by a Pedo Orthopedic specialist and Pediatrician and the Belghton test was scored as an assessment tool. Furthermore, they were reffered to a specialized Pediatric Physiotherapist for clinical evaluation. Children with Symptomatic Flexible Flat Foot (SFFF) and positive Belghton test were treated with customized orthotic insoles. Recommendations were made to use orthoses on everyday basis while weight-baring and documentation of the symptomatology by the scientific team and the parents would be reviewed after six months' use.

It is a cohort study where collective results cannot be supported because of the small sample and the absence of control group. This is an inherent limitation which concerns all such studies and it emphasizes the need for more extended research. Another limitation factor is the worldwide truancy of standardized assessment tools of the deviations of lower limbs.

Data used in this study were retrieved from electronic databases (Cochrane, Medline, AMED, EMBASE, CINHAL, SportDiscus, Scopus, Physiotherapy-PEDro, PUBMED) from January 2019 to May 2020. Key words were used: Pesplanus, Pesplanus, Planovalgus, flat feet (flat foot), low arch, orthotics, insoles, treatment of PFF, non surgical intervention, child, adolescent. Electronic research was restricted to humans, english language and 0 - 18 years of age.

\section{Statistical Analysis}

The advanced statistical software GraphPad Prism version 7.00 for Windows (GraphPad Software, La Jolla California USA, www.graphpad.com) was used for the statistical evaluation of patients outcome before and after treatment, applying Fisher's exact test for the comparison of categorical variables, as it is more accurate for small sample sizes. SPSS ver. 25.0 (IBM SPSS, Armonk, NY, USA) was used for Cramer's V tests, in order to evaluate possible significant association of outcomes after treatment. Statistical significance threshold was set at 0.05 .

\section{Results}

Twenty children with mobility difficulties were evaluated before and after a six-month treatment with orthopedics, regarding pain, post-game pain, fatigue during game, balance impairment, gait deviations, falls, clumsiness, activity avoidance and worn soles. A great statistical difference was observed for both pain and post-game pain with only $30 \%$ of patients still feeling pain after treatment. Statistically significant improvement was also recorded for gait deviations, clumsiness and worn soles, and a slightly one for balance impairment and falls.

Regarding pain all of the subjects tested complained about pain before treatment. After a six-month treatment the percentage decreased to $30 \%$. Fisher's exact test indicated a strong statistical significance in pain reduction with a $\mathrm{p}$ value $<0.0001$. The same rates were also observed for post-game pain. Statistically significant improvement was also recorded for gait deviations, as the num- 

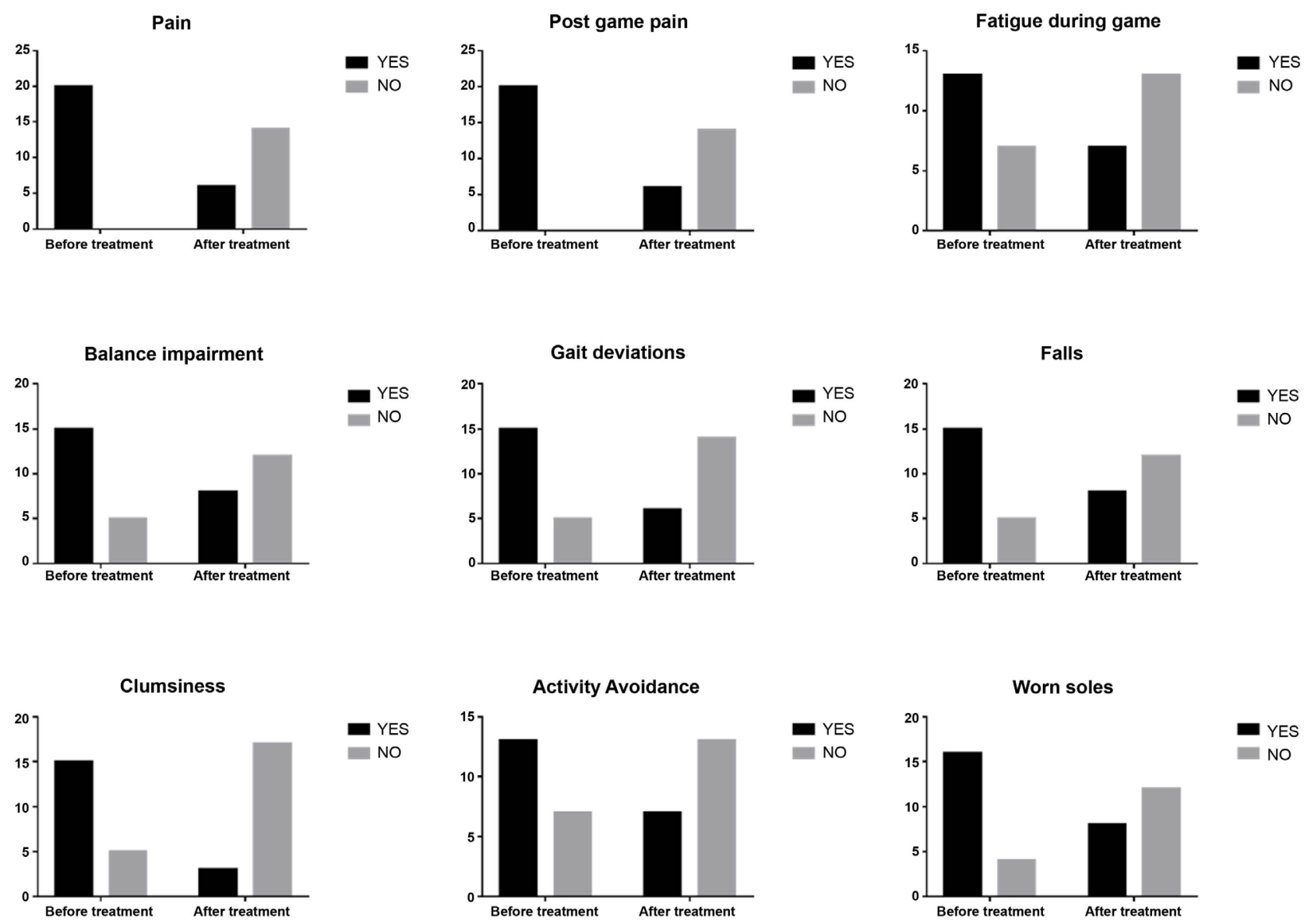

Figure 1. Graphical illustration of patients' answers regarding all parameters tested before and after treatment. 
Table 1. Patients' outcomes before and after treatment.

\begin{tabular}{cccc}
\hline Outcome & Before treatment $(\mathrm{n}=20)$ & After treatment $(\mathrm{n}=20)$ & pvalue \\
\hline Pain (n, \%) & $20(100 \%)$ & $6(30 \%)$ & $<0.0001$ \\
Post-game pain (n, \%) & $20(100 \%)$ & $6(30 \%)$ & $<0.0001$ \\
Fatigue during game (n, \%) & $13(65 \%)$ & $7(35 \%)$ & 0.113 \\
Balance impairment (n, \%) & $15(75 \%)$ & $8(40 \%)$ & 0.053 \\
Gait deviations (n, \%) & $15(75 \%)$ & $6(30 \%)$ & 0.01 \\
Falls (n, \%) & $15(75 \%)$ & $8(40 \%)$ & 0.053 \\
Clumsiness (n, \%) & $15(75 \%)$ & $3(15 \%)$ & 0.0003 \\
Activity Avoidance (n, \%) & $13(65 \%)$ & $7(35 \%)$ & 0.113 \\
Worn soles (n, \%) & $16(80 \%)$ & $8(40 \%)$ & 0.023 \\
\hline
\end{tabular}

\section{Discussion}

Aim of this study is to collect information on the recent literature and to investigate through clinical assessment and parental observations the effectiveness of the use of foot orthotics by children with symptomatic flexible flat foot (SFFF). More specifically, data where retrieved and analised through a form filled by the medical team and the parents, before and after a six months' use of orthotics of the lower limb in children with symptomatic flatfoot.

Study results indicate that foot orthotics (FOs) appear to have a positive impact on the advance of pain, post game pain, gait deviations and worn insoles. A large and statistically significant percentage has a considerable amelioration of pain on a daily basis as well as distinct improvement of post game pain simply by using lower limb orthotics. Statistically significant were the results regarding improvement of gait deviations, clumsiness and worn insoles, as well as a slightly less balance impairment and falls. No differentiation in fatigue during game and activity avoidance was reported.

Literature research findings are in accordance with the outcomes of our study of a pediatric population establishing alterations by using orthotics [17]-[22]. In particular, Whitford and Esterman have compared a group of custom-made versus ready-made orthoses concluding that there is improvement in pain relief and function after 12 months, but with no statistically significant difference between the two groups (19). Lee, H.-J et al. have demonstrated that short-term use of adaptive orthotics of the foot has notably eliminated pain and balance impairment in children with symptomatic flexible flat feet (SFFF) [20].

Powell et al., in an attempt not to surgically intervene in pediatric symptomatic flexible flat feet (SFFF) with symptoms of arthritis and pain of the lower extremity, has reached the conclusion that by using adaptive orthotics we achieve drastic pain relief [21]. A recent systematic review of orthotics in pediatric patients (2018) demonstrates that plethora of evidence supports that orthotics (FOs) can have a positive effect on pain, posture of feet, gait, structural and 
functional features of the foot [22].

Heterogenous studies on symptomatic flexible flat foot (SFFF) report limited evidence on the use of orthotics in pediatric populations [23] [24]. Results of two studies show that pediatric flatfoot (PFF) does not have an effect on the ability to move, exercise or permanently avoid activity [25] [26]. Another study indicates that pes planus does not need any intervention unless symptoms are induced [27]. In cases where malfunctions or diverse symptoms are confirmed, such as pain, impairment of the ability to exercise, gait deviations or fatigue, the patient should be evaluated and interventions should be discussed on a new basis [28] [29]. Mac Kenzie et al. (2012) could not definitely deduce that orthotics have statistically significant positive results when treating pediatric pes planus [30].

Thus, current evidence throughout the literature lacks uniformity in terms of consensus on treatment of pediatric pes planus or symptomatic flexible flat foot (SFFF) with orthotics. Acknowledgements of the lack of clarity of evidence of the present databases should be made, in order to compose further research more precise and standardized on a larger pediatric population to properly justify the use of orthoses (FOs).

It should be mentioned that the present study appears to have several restrictions. In particular, it is consisted of a single group of "patients" and no extensive results should be reached, given the small sample and the absence of a control group, which is an intrinsic defect of such studies.

A necessity is set to conduct randomized control studies for safe deductions on the use of foot orthoses (FOs).

Finally, it should be highlighted that future studies will benefit from a wide pediatric sample and will focus on the development of standardized diagnostic criteria regarding the use of custom-made foot orthotics (FOs).

\section{Conclusion}

Despite various diversities in literature references regarding accurate documentation of the efficacy of foot orthotics (FOs), there are emerging positive facts in our research that claim improvement after using adaptive orthotics of the lower extremities in parameters such as: pain, post game pain, gait deviations and worn insoles. Apart from clinical evaluation of the symptomatology, facts retrieved through the questionnaire filled by the parents reinforce the positive outcome after a six month's use of foot orthotics (FOs). Due to the small sample used in the research, the effectiveness of foot orthotics (FOs) in symptomatic pediatric flexible flat feet (SFFF) still remains to be confirmed. Although there are limitations in methodology and application, foot orthotics (FOs) appear to have a great potential as a treatment for symptomatic pediatric flexible flat foot (SFFF). Thorough comprehension of the literature evidence, as well as composition of supplementary studies of larger pediatric populations is essential in order to reach a consensus on the use of foot orthotics (FOs) by children with symptomatic flexible flat foot (SFFF). 


\section{Conflicts of Interest}

The authors declare no conflicts of interest regarding the publication of this paper.

\section{References}

[1] Pauk, J. and Griskevicius, J. (2011) Ground Reaction Force and Support Moment in Typical and Flat-Feet Children. Mechanics, 17, 93-96.

https://doi.org/10.5755/j01.mech.17.1.209

[2] Foot Health Facts. The Official Consumer Website of American College of Foot and Ankle Surgeons. http://www.foothealthfacts.org/footankleinfo/pediatric-flatfoot.htm

[3] Benedetti, M.G., Ceccarelli, F., Berti, L., Luciani, D., Catani, F., Boschi, M., et al. (2011) Diagnosis of Flexible Flatfoot in Children: A Systematic Clinical Approach. Orthopedics, 34, 94. https://doi.org/10.3928/01477447-20101221-04

[4] Caselli, M.A., Sobel, E. and McHale, K.A. (1998) Pedal Manifestations of Musculoskeletal Disease in Children. Clinics in Podiatric Medicine and Surgery, 15, 481-497.

[5] Harris, E.J. (2000) The Oblique Talus Deformity. What Is It, and What Is Its Clinical Significance in the Scheme of Pronatory Deformities? Clinics in Podiatric Medicine and Surgery, 17, 419-442.

[6] Harris, E.J., Vanore, J.V., Thomas, J.L., et al. (2004) Diagnosis and Treatment of Pediatric Flatfoot. The Journal of Foot and Ankle Surgery, 43, 341-373. https://doi.org/10.1053/j.jfas.2004.09.013

[7] Fabry, G. (2010) Clinical Practice. Static, Axial, and Rotational Deformities of the Lower Extremities in Children. European Journal of Pediatrics, 169, 529-534. https://doi.org/10.1007/s00431-009-1122-x

[8] Yagerman, S.E., Cross, M.B., Positano, R. and Doyle, S.M. (2011) Evaluation and Treatment of Symptomatic Pes Planus. Current Opinion in Pediatrics, 23, 60-67.

[9] Nemeth, B. (2011) The Diagnosis and Management of Common Childhood Orthopedic Disorders. Current Problems in Pediatric and Adolescent Health Care, 41, 2-28. https://doi.org/10.1016/j.cppeds.2010.10.004

[10] Houghton, K.M. (2008) Review for the Generalist: Evaluation of Pediatric Foot and ankle Pain. Pediatric Rheumatology, 6, Article No. 6. https://doi.org/10.1186/1546-0096-6-6

[11] Ferciot, C.F. (1972) The Etiology of Developmental Flatfoot. Clinical Orthopaedics and Related Research, 85, 7-10.

[12] Basmajian, J.V. and Stecko, G. (1963) The Role of Muscles in Arch Support of the Foot. Journal of Bone and Joint Surgery, 45, 1184-1190.

[13] Carter, C. and Wilkinson, J. (1964) Persistent Joint Laxity and Congenital Dislocation of the Hip. The Journal of Bone and Joint Surgery, 46-B, 40-45. https://doi.org/10.1302/0301-620X.46B1.40

[14] Hicks, J.H. (1954) The Mechanics of the Foot. II. The Plantar Aponeurosis and the Arch. Journal of Anatomy, 88, 25-30.

[15] Evans, A.M. (2008) The Flat-Footed Child-To Treat or Not to Treat. What Is the Clinician to Do? Journal of the American Podiatric Medical Association, 98, 386-393. https://doi.org/10.7547/0980386

[16] Stavlas, P., Grivas, T.B., Michas, C., Vasiliadis, E. and Polyzois, V. (2005) The Evolution of Foot Morphology in Children between 6 and 17 Years of Age: A CrossSectional Study Based on Footprints in a Mediterranean Population. The Journal of 
Foot \& Ankle Surgery, 44, 424-428. https://doi.org/10.1053/j.jfas.2005.07.023

[17] Chen, K.-C., Tung, L.-C., Tung, C.-H., Yeh, C.-J., Yang, J.-F. and Wang, C.-H. (2014) An Investigation of the Factors Affecting Flatfoot in Children with Delayed Motor Development. Research in Developmental Disabilities, 35, 639-645. https://doi.org/10.1016/j.ridd.2013.12.012

[18] Dunn, J., Link, C., Felson, D., Crincoli, M., Keysor, J. and McKinlay, J. (2004) Prevalence of Foot and Ankle Conditions in a Multiethnic Community Sample of Older Adults. American Journal of Epidemiology, 159, 491-498. https://doi.org/10.1093/aje/kwh071

[19] Whitford, D. and Esterman, A. (2007) A Randomized Controlled Trial of Two Types of In-Shoe Orthoses in Children with Flexible Excess Pronation of the Feet. Foot \& Ankle International, 28, 715-23. https://doi.org/10.3113/FAI.2007.0715

[20] Lee, H.-J., Lim, K.-B., Yoo, J., Yoon, S.-W., Yun, H.-J., \& Jeong, T.-H. (2015) Effect of Custom-Molded Foot Orthoses on Foot Pain and Balance in Children with Symptomatic Flexible Flat Feet. Annals of Rehabilitation Medicine, 39, 905-913. https://doi.org/10.5535/arm.2015.39.6.905

[21] Powell, M., Seid, M. and Szer, I.S. (2005) Efficacy of Custom Foot Orthotics in Improving Pain and Functional Status in Children with Juvenile Idiopathic Arthritis: A Randomized Trial. The Journal of Rheumatology, 32, 943-950.

[22] Dars, S., Uden, H., Banwell, H.A. and Kumar, S (2018) The Effectiveness of NonSurgical Intervention (Foot Orthoses) for Paediatric Flexible Pes Planus: A Systematic Review: Update. PLOS ONE, 13, e0193060.

https://doi.org/10.1371/journal.pone.0193060

[23] Rome, K., Ashford, R.L. and Evans, A. (2010) Non-Surgical Interventions for Paediatric Pes Planus. Cochrane Database of Systematic Reviews, No. 7, CD006311. https://doi.org/10.1002/14651858.CD006311.pub2

[24] Evans, A.M. and Rome, K. (2011) A Cochrane Review of the Evidence for NonSurgical Interventions for Flexible Pediatric Flat Feet. European Journal of Physical and Rehabilitation Medicine, 47, 69-89.

[25] Pfeiffer, M., Kotz, R., Ledl, T., Hauser, G. and Sluga, M. (2006) Prevalence of Flat Foot in Preschool-Aged Children. Pediatrics, 118, 634-639.

https://doi.org/10.1542/peds.2005-2126

[26] Tudor, A., Ruzic, L., Sestan, B., Sirola, L. and Prpić, T. (2009) Flat-Footedness Is Not a Disadvantage for Athletic Performance in Children Aged 11 to 15 Years. Pediatrics, 123, e386-e392. https://doi.org/10.1542/peds.2008-2262

[27] Yan, G.S., Yang, Z., Lu, M., Zhang, J.L., Zhu, Z.H. and Guo, Y. (2013) Relationship between Symptoms and Weight-Bearing Radiographic Parameters of Idiopathic Flexible Flatfoot in Children. Chinese Medical Journal, 126, 2029-2033.

[28] Evans, A.M., Rome, K. and Peet, L. (2012) The Foot Posture Index, Ankle Lunge Test, Beighton Scale and the Lower Limb Assessment Score in Healthy Children: A Reliability Study. Journal of Foot and Ankle Research, 5, Article No. 1. https://doi.org/10.1186/1757-1146-5-1

[29] Krul, M., Van Der Wouden, J.C., Schellevis, F.G., Van Suijlekom-Smit, L.W. and Koes, B.W. (2009) Foot Problems in Children Presented to the Family Physician: A Comparison between 1987 and 2001. Family Practice, 26,174-179. https://doi.org/10.1093/fampra/cmp018

[30] MacKenzie, A., Rome, K. and Evans, A.M. (2012) The Efficacy of Nonsurgical Interventions for Pediatric Flexible Flat Foot: A Critical Review. Journal of Pediatric Orthopaedics, 32, 830-834. https://doi.org/10.1097/BPO.0b013e3182648c95 